\title{
Initiating a watch list for Ebola virus antibody escape mutations
}

Craig R Miller, Erin L Johnson, Aran Z Burke, Kyle P Martin, Tanya A Miura, Holly A Wichman, Celeste J Brown, F Marty Ytreberg

The 2014 Ebola virus (EBOV) outbreak in West Africa is the largest in recorded history and resulted in over 11,000 deaths. It is essential that strategies for treatment and containment be developed to avoid future epidemics of this magnitude. With development of vaccines and antibody-based therapies using the envelope glycoprotein (GP) of the 1976 Mayinga strain, one important strategy is to anticipate how the evolution of EBOV might compromise these efforts. In this study we have initiated a watch list of potential antibody escape mutations of EBOV by modeling interactions between GP and the antibody KZ52. The watch list was generated using molecular modeling to estimate stability changes due to mutation. Every possible mutation of GP was considered and the list was generated from those that are predicted to disrupt GP-KZ52 binding but not to disrupt the ability of GP to fold and to form trimers. The resulting watch list contains 34 mutations (one of which has already been seen in humans) at six sites in the GP2 subunit. Should mutations from the watch list appear and spread during an epidemic, it warrants attention as these mutations may reflect an evolutionary response from the virus that could reduce the effectiveness of interventions such as vaccination. However, this watch list is incomplete and emphasizes the need for more experimental structures of EBOV interacting with antibodies in order to expand the watch list to other epitopes. We hope that this work provokes experimental research on evolutionary escape in both Ebola and other viral pathogens. 


\title{
Initiating a Watch List for Ebola Virus Antibody Escape Mutations
}

\author{
Craig R. Miller ${ }^{1,2,3,5}$, Erin L. Johnson ${ }^{3}$, Aran Z. Burke ${ }^{3,4}$, Kyle P. Martin ${ }^{3,4}$, \\ Tanya A. Miura ${ }^{1,3}$, Holly A. Wichman ${ }^{1,3,5}$, Celeste J. Brown ${ }^{1,3,5}$, and F. \\ Marty Ytreberg ${ }^{3,4,5, *}$

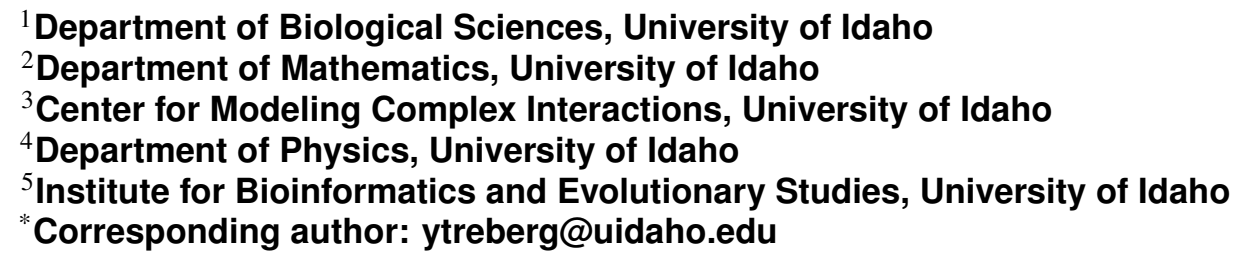

12 ABSTRACT

The 2014 Ebola virus (EBOV) outbreak in West Africa is the largest in recorded history and resulted in over 11,000 deaths. It is essential that strategies for treatment and containment be developed to avoid future epidemics of this magnitude. With development of vaccines and antibody-based therapies using the envelope glycoprotein (GP) of the 1976 Mayinga strain, one important strategy is to anticipate how the evolution of EBOV might compromise these efforts. In this study we have initiated a watch list of potential antibody escape mutations of EBOV by modeling interactions between GP and the antibody KZ52. The watch list was generated using molecular modeling to estimate stability changes due to mutation. Every possible mutation of GP was considered and the list was generated from those that are predicted to disrupt GP-KZ52 binding but not to disrupt the ability of GP to fold and to form trimers. The resulting watch list contains 34 mutations (one of which has already been seen in humans) at six sites in the GP2 subunit. Should mutations from the watch list appear and spread during an epidemic, it warrants attention as these mutations may reflect an evolutionary response from the virus that could reduce the effectiveness of interventions such as vaccination. However, this watch list is incomplete and emphasizes the need for more experimental structures of EBOV interacting with antibodies in order to expand the watch list to other epitopes. We hope that this work provokes experimental research on evolutionary escape in both Ebola and other viral pathogens.

Keywords: FoldX, Molecular Dynamics, Protein Stability, Escape Mutations

\section{INTRODUCTION}

With nearly 30,000 confirmed cases and over 11,000 deaths, the recent Ebola virus (EBOV) epidemic in West Africa has dwarfed all recorded outbreaks of the disease (cdc, 2015). Now that the 2014 outbreak appears to be waning it is critical to develop strategies for treatment and containment to avoid future epidemics of this magnitude. One important strategy is the development of vaccines. Two vaccines that express the EBOV envelope glycoprotein (GP) from the 1976 Mayinga strain are in phase III clinical trials: rVSV-ZEBOV and ChAd3-ZEBOV (Garbutt et al., 2004; Stanley et al., 2014; Marzi et al., 2013). Early reports suggest that rVSV-ZEBOV is highly effective at preventing EBOV infection (Henao-Restrepo et al., 2015). A related strategy is antibody-based therapeutics. For example, ZMapp has been shown to be effective in treating non-human primates and has been used to treat small numbers of humans with Ebola (Qiu et al., 2014; Borio et al., 2015). The monoclonal antibodies in ZMapp were generated by vaccination of mice with GP from the 1976 Mayinga strain (Wilson et al., 2000; Qiu et al., 2011, 2014).

A key course of action to prepare for future EBOV outbreaks is to anticipate how the evolution of antibody escape mutants in the virus might compromise treatment efforts. Antibody escape mutants have arisen in the laboratory when recombinant vesicular stomatitis viruses expressing the GP protein of EBOV or Marburg virus were grown in the presence of anti-GP antibodies (Kajihara et al., 2013). In that study a single amino acid substitution conferred viral resistance to the antibodies. Similarily, a 
single amino acid change in GP of the EBOV Kikwit 95 strain in a macaque treated with monoclonal antibodies resulted in fatal infection (Qiu et al., 2012). Mutational changes in GP have also been found to impact immune responses to the virus; substitutions at $\mathrm{N}$-linked glycosylation sites can alter antigenicity and immunogenicity, in some cases preventing binding to the KZ52 antibody (Dowling et al., 2007; Lennemann et al., 2015). Antibody escape mutants are also known in influenza A, HIV 1, measles and respiratory syncytial virus infections (Smith et al., 2004; Geiß and Dietrich, 2014; Schrag et al., 1999; Zhao et al., 2006).

Sequencing studies have shown that there is a high level of genetic variation in EBOV and that GP has the largest variation among EBOV proteins (Gire et al., 2014; Tong et al., 2015; Park et al., 2015). As of August 2015, sequences from the 2014 outbreak show that 106 of the 676 sites in GP experienced a mutation and the strains differ from the 1976 Mayinga strain used in developing interventions by an average of 20.2 nucleotide changes. Thus, there is a very real possibility of antibody escape mutants arising in EBOV GP. A recent study found that none of the genetic changes have altered the function of the virus (Olabode et al., 2015). However, they did not consider interactions with antibodies or implications of unobserved mutations.

The purpose of this study is to initiate a watch list of potential antibody escape mutants for the EBOV GP. We focus on the KZ52 antibody as it is one of the few with an available structure bound to EBOV GP. KZ52 has virus neutralization activity in vitro and protects guinea pigs from EBOV disease (Maruyama et al., 1999; Parren et al., 2002). Although KZ52 does not protect non-human primates from EBOV disease (Oswald et al., 2007), it was originally isolated from the blood of human EBOV survivors (Maruyama et al., 1999). Using the experimental structure of the Zaire EBOV GP bound to antibody KZ52 (Fig. 1) (Lee et al., 2008), we performed molecular modeling to estimate the folding and binding stabilities for every possible amino acid mutation of GP. Our approach is general and could be applied to other EBOV epitopes, or other viruses, as experimental structures become available. We emphasize from the outset that this is an in silico study aimed at identifying mutations with an increased risk of escaping immune response; our intention is to provoke experimental research on evolutionary escape in both Ebola and other viral pathogens. 

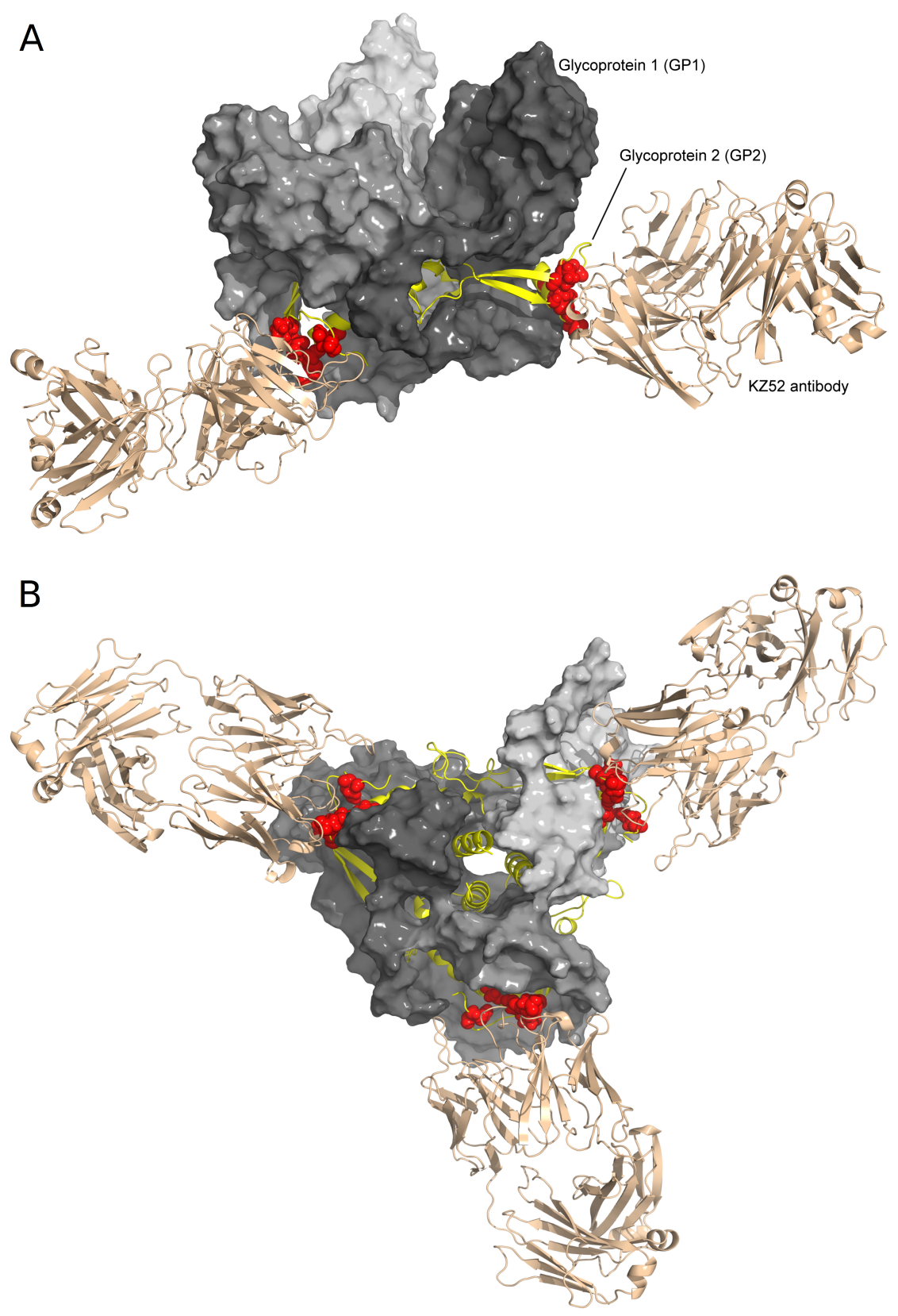

Figure 1. Structure of Ebola glycoprotein trimer in complex with the KZ52 antibody as viewed from the side (A) and the bottom (B). GP1 is in gray, GP2 is in yellow and the structure is after $10 \mathrm{~ns}$ of MD simulation. The six watch list sites that are predicted to contain antibody escape mutants are shown as red spheres and are all located in GP2 (Table 2, Fig 2.)

\section{METHODS}

\section{Overview}

To initiate a watch list for the Ebola virus (EBOV) glycoprotein (GP) it is necessary to determine how amino acid mutations modify stabilities for GP folding, forming a trimer and binding to the KZ52 antibody. That is, we need to calculate $\Delta \Delta G$ values for binding and folding. Ideally, these calculations would be performed using a statistical-mechanics-based method such as we have done previously (Lee et al., 2011; Zhan and Ytreberg, 2015). However, such methods are computationally expensive and are not feasible for the current study where it was necessary to calculate 25,840 values of $\Delta \Delta G$ (340 residues $\times 19$ 
possible mutations to other residues $\times 4$ types of stability calculations). Instead, we decided to use a semi-empirical method for calculating $\Delta \Delta G$ values. Because online-only software was not practical given the large number of mutations, we chose to use the software FoldX (Schymkowitz et al., 2005; Guerois et al., 2002). FoldX can be run in parallel on a computer cluster since the binary is available.

We hypothesized that because protein structures are not static, improvements in $\Delta \Delta G$ estimation might be achieved by using molecular dynamics simulation to sample the configurational space for the proteins and then analyze snapshots from these simulations in FoldX. We selected 20 test systems (10 folding and 10 binding) to assess whether this strategy improves estimation of experimental stability data. In the Supplemental Materials, we describe our criteria for selecting test systems and then show that using 100 molecular dynamics snapshots and averaging the FoldX results provides more accurate estimates of $\Delta \Delta G$ as compared to using FoldX on a single experimental structure. The molecular dynamics plus FoldX methodology we used on the test systems was identically applied to the Ebola system. After explaining how structures were prepared and arranged, we describe this methodology in the subsections below.

\section{Stability estimation Structure preparation}

Preparation of the test system structures is described in the Supplemental Materials. For EBOV GP, the amino acid sequence was based on the 1976 Mayinga strain first obtained from GenBank accession number AF086833. We downloaded PDB accession number 3CSY as our template structure. The file 3 csy.pdb was modified to remove all but one copy each of GP1, GP2, antibody light chain and antibody heavy chain (one third of the GP-KZ52 trimeric complex). SWISS-MODEL was then used to generate structures for each of the four chains using 3csy.pdb as a template Arnold et al. (2005). The experimental structure 3csy has missing residues 190-213 that are predicted to be intrinsically disordered but SWISSMODEL incorrectly generated helical structures for these residues. Thus, we removed residues 190-213 from the SWISS-MODEL structure and used MODELLER to rebuild the coordinates of the missing residues Sali and Blundell (1993). The resulting structure had no secondary structure content in residues 190-213. The full trimeric complex was then created using the symexp command in PyMOL. The final trimer structure (see Fig. 1) contains three copies each of residues 32-276 for GP1, residues 503-597 for GP2, residues 1-225 for KZ52 heavy chain and residues 1-216 for KZ52 light chain.

\section{System configuration}

Arrangement of the test systems is described in the Supplemental Materials. EBOV GP was configured as four systems: (i) unbound GP1, (ii) unbound GP2, (iii) trimer consisting of three copies of GP1 and GP2 and (iv) antibody complex consisting of three copies each of GP1, GP2 and the KZ52 antibody. Snapshots from systems (i) and (ii) were analyzed as described next to estimate mutational effects on folding stability of the unbound proteins GP1 and GP2, respectively. Snapshots from (iii) were used to estimate the affinity of GP1-GP2 (dimer bind). This was done by calculating the affinity for all three copies of GP1 binding to GP2 and then dividing this value by three. Snapshots from (iii) were also used to estimate the affinity for GP1-GP2 dimers binding to one another (trimer bind). This was done by calculating the affinity for one GP1-GP2 dimer binding to the other two dimers. Finally, snapshots from (iv) were used to estimate the GP-KZ52 affinity by calculating the affinity of all of the GP1-GP2 dimers to their corresponding KZ52 antibodies and dividing this value by three.

\section{Molecular dynamics simulations}

The software package GROMACS 5.0.3 was used for all MD simulations with the Charmm22* forcefield (Hess et al., 2008). The system was placed in a dodecahedral box of TIP3P water and given neutral charge by adding $\mathrm{Na}+$ and $\mathrm{Cl}$ - ions at a concentration of $0.15 \mathrm{~mol} / \mathrm{L}$. Each system was then minimized using steepest decent for 1000 steps. To allow for some equilibration of the water around the proteins each system was then simulated for $1 \mathrm{~ns}$ with the positions of all heavy atoms in the complex harmonically restrained, and then simulated for another $1 \mathrm{~ns}$ with no restraints. During the restrained simulations the temperature of the system was increased linearly from $100 \mathrm{~K}$ to $300 \mathrm{~K}$ for the test systems and to 310 $\mathrm{K}$ for the EBOV GP systems and the pressure was maintained at $1 \mathrm{~atm}$ using the Berendsen algorithm. Production simulations for each system were then carried out for $100 \mathrm{~ns}$ with pressure maintained using Parrinello-Rahman coupling. For all simulations the LINCS algorithm was used to constrain all bonds to their ideal lengths and virtual sites were used allowing the use of a $5 \mathrm{fs}$ timestep. The temperature was controlled using the v-rescale option. Particle mesh Ewald was used for electrostatics with a real-space 
cutoff of $1.2 \mathrm{~nm}$. Van der Waals interactions were cut off at $1.2 \mathrm{~nm}$ with the Potential-shift-Verlet method for smoothing interactions. During the $100 \mathrm{~ns}$ production simulation snapshots were saved every $100 \mathrm{ps}$ giving 100 snapshots.

\section{FoldX}

Each of the 100 snapshots captured during MD simulations was then analyzed using FoldX (Schymkowitz et al., 2005; Guerois et al., 2002). We initially minimize structures six times in succession using the RepairPDB command to obtain convergence of the potential energy. All single amino acid mutations were then generated using BuildModel. Finally, protein folding stabilities were estimated using Stability on the monomer structures and binding stabilities were estimated using AnalyseComplex on the protein complexes. For each mutation we then estimated $\Delta \Delta G$ by averaging across all 100 individual snapshots estimates.

\section{Thresholds for functionality and antibody disruption}

To define the range of stability change where the GP protein is likely to remain functional, we began by noting that in previous work on the bacteriophage $\phi X 174$ (Miller et al., 2014), 77 of 79 (97.5\%) of observed functional mutations have estimated stability effects on both folding and binding in the range $-2.5<\Delta \Delta G<2.5 \mathrm{kcal} / \mathrm{mol}$. The large amount of available Ebola sequences allows us to survey a set of presumably functional mutations in Ebola and ask how many of these are categorized as functional vs non-functional using this preliminary criteria. We downloaded 922 sequences from the NCBI Virus Variation Ebolavirus Database on August 20, 2015 (Brister et al., 2013; ncb, 2015) (Species = Zaire ebolavirus, Host=Any, Region=Any, Genome Region=Spike glycoprotein). To this set we appended 39 sequences from (Leroy et al., 2004) and (Wittmann et al., 2007) along with the two escape mutations described in (Qiu et al., 2012). We compared all 962 sequences to our reference sequence, GenBank Accession AF086833, and thereby identified 41 mutational differences (Table 1) within the structured regions modeled here. Four of the 41 mutations $(9.8 \%)$ have a functional stability effect (i.e., $\Delta \Delta G$ for monomer folding, dimer binding or trimer binding) that falls outside the \pm 2.5 zone. Because our objective is to limit the rate of false exclusions to $\leq 5 \%$, we expanded the functional zone to \pm 3.0 . This shifts two of the mutations back into the functional zone, leaving 2 of $41(4.9 \%)$ predicted to be non-functional.

It is worth noting that the observed incidence of two false exclusions in a sample of 41 is consistent with our method having predictive power to distinguish functional from non-functional mutations. Of the 6460 possible mutations for GP, our method categorizes $5303(82.1 \%)$ as functional and $1157(17.9 \%)$ as non-functional. If our method lacked predictive power we would expect a random sample of 41 mutations to contain 33.7 functional and 7.3 non-functional mutants. The binomial probability that such a random sample would contain $\leq 2$ non-functional proteins by chance is 0.018 . Unfortunately, because we lack a list of known non-functional mutations, we cannot perform the converse test and ask what proportion of non-functional mutations does our method correctly identify as such.

How sensitive is the size of the watch list to the rate of false exclusions? The following argument suggests that even if the false exclusion rate could be reduced to zero, it would have a very small effect on the watch list. The application of a functional zone bewteen $\pm 3.0 \mathrm{kcal} / \mathrm{mol}$ along with an antibody disruption criteria of $\Delta \Delta G>2.0 \mathrm{kcal} / \mathrm{mol}$ leads to a watch list of 34 mutations. Of the 6460 possible mutations, our method categorizes 1157 as non-functional. If $5 \%$ of these are actually functional, it suggests that we have omitted approximately $6460(0.05)=58$ mutations from the set of functional mutations. However, very few of these would likely disrupt antibody binding. Among all 6460 mutations, 66 (or $\approx 1 \%$ ) are identified as disrupting antibody binding. Assuming false exclusion is independent of antibody disruption, we would expect that $58(0.01)=0.6$, or less than one mutation being falsely omitted from the watch list.

\section{RESULTS AND DISCUSSION}

We identified potential antibody escape mutations for the watch list by considering every possible GP mutation and finding those that disrupt binding between GP and KZ52 but do not disrupt the ability of GP to fold and form a complex. The GP protein is cleaved into two subunits, GP1 and GP2, and the final structure is a trimer consisting of three GP1-GP2 dimers (Fig. 1). We used a combination of molecular dynamics and FoldX (Schymkowitz et al., 2005; Guerois et al., 2002) because preliminary analysis of 20 
test systems showed that combining these methods improved our ability to predict experimental results (see Supplemental Materials). To our knowledge this method has not been used in previous studies.

Our conceptual approach to creating a watch list is to identify mutations that are both functional and disrupt antibody binding. We therefore sought to remove mutations that are non-functional and, from those that remain, identify the ones that disrupt antibody binding. The function of GP is to mediate viral entry into the cell. There are multiple ways mutation can disrupt this function. For example, studies have shown that mutations in GP can reduce infectivity (Ito et al., 1999; Watanabe et al., 2000; Davidson et al., 2015), transduction and host cell binding (Dube et al., 2009; Brindley et al., 2007). Another way to be non-functional is for a mutation to render GP unable to fold and bind together to form a stable complex. Here we focus on this stability aspect of functionality and remove those mutations our model predicts will not fold or form a complex. It is important to appreciate that our approach is conservatively inclusive: if we could remove all non-functional mutations instead of the subset identified as unstable, the watch list would be reduced in size.

Identifying mutations that disrupt antibody binding but not the ability to fold and bind into a functional complex requires defining thresholds on changes in stability $(\Delta \Delta G)$ for both criteria. These criteria should be conservative to reduce exclusion of mutations that could compromise treatment efficacy from the watch list. For functionality, previous work on a coat protein in a different virus (Miller et al., 2014) indicated that the stability effect of virtually all observed mutations is in the range of $-2.5<\Delta \Delta G<2.5$ $\mathrm{kcal} / \mathrm{mol}$. To determine if those criteria also hold for EBOV GP, we compared 963 available sequences of GP, identified 41 mutations in the structured regions that have arisen in natural or lab populations, and found that four of the $41(9.8 \%)$ were classified as non-functional. To be conservative, we expanded the functional zone to $-3.0<\Delta \Delta G<3.0 \mathrm{kcal} / \mathrm{mol}$. This functional threshold is more inclusive and reduces our error rate to below 5\%: two of the 41 mutations (4.9\%) are falsely classified as non-functional (Table 1). As we reason in the Methods, even if the false exclusion rate could be driven to zero, we expect it would change our watch list very little. For disruption of antibody binding, we used a threshold of $\Delta \Delta G>2.0 \mathrm{kcal} / \mathrm{mol}$. This was based on refining our preliminary threshold by $0.5 \mathrm{kcal} / \mathrm{mol}$, but in the opposite direction so as to be more inclusive. The implications of this threshold choice and alternatives to it will be discussed below.

Figure 2 provides a graphical illustration of how mutations were selected to be on the watch list. The maximum functional stability for all mutations is plotted against the corresponding change in the antibody binding affinity. The 34 mutations in the lower right quadrant are those that belong on the watch list since they are classified as both functional and disruptive to antibody binding. The specific mutations on the watch list are given in Table 2. If any of the mutations in this table appear in a real population, it indicates an increased risk of escaping the normal immune response. One of these mutations (N550K) has already appeared in humans thought to have been infected by gorillas in Central Africa between 2001-2003 (Leroy et al., 2004). This mutation is present in all sequenced isolates from that outbreak.

The ultimate goal is to develop a complete watch list. This is an initial step in that process.

If mutations on the watch list increase in frequency in an immunized population, it would suggest that the virus is evolving to escape immune response.

In contrast to constructing a simple list of all possible mutations near an epitope, the watch list in Table 2 is quite specific. The 34 watch list mutations are concentrated at just six residues and all of these lie at the interface between GP2 and KZ52, as one might intuitively expect from the structure (Fig. 1). Yet, most mutations of GP sites that are within four angstroms of the KZ52 antibody are not predicted to disrupt antibody binding. Only six of the 23 (26\%) interface sites and 34 of $437(7.8 \%)$ of the possible mutations at these sites are on the watch list.

In order to facilitate use of other possible criteria and/or thresholds, the Supplemental Materials includes a sortable and searchable spreadsheet with all 6460 mutations of GP. We provide this spreadsheet because we recognize that the relationship between antibody binding affinity and the ability of the antibody to neutralize EBOV is not well understood. Work in influenza suggests that as affinity decreases, the ability of an antibody to neutralize a virus decreases rapidly and in a non-linear fashion (Kostolanskỳ et al., 2000). This makes intuitive sense because the relationship between the change in affinity and the change in the ratio of bound to unbound antibody is nonlinear; $\Delta \Delta G$ values of $1.0,1.5$ and $2.0 \mathrm{kcal} / \mathrm{mol}$ correspond to changing the ratio from 19 to 8.1 to $3.5 \%$ of its original value. The size of the watch list depends on how we define the threshold for antibody binding (Fig. 2). If the threshold is lowered from 2.0 to 1.5 to 1.0 , the watch list grows from 34 to 49 to 73 mutations. This highlights the need for more 
Table 1. Model predicted effects on stability of 41 observed mutations.

\begin{tabular}{|c|c|c|c|c|}
\hline Mutation & Antibody Bind $^{a}$ & Dimer Bind $^{b}$ & Trimer Bind $^{c}$ & Monomer Fold $^{d}$ \\
\hline N107D & 0.00 & -0.09 & 0.00 & 1.31 \\
\hline L111F & 0.00 & 0.00 & 0.00 & 2.31 \\
\hline $\mathrm{I} 129 \mathrm{~V}$ & 0.00 & 0.05 & 0.02 & 0.77 \\
\hline D150A & 0.00 & 0.00 & 0.01 & 0.6 \\
\hline D163N & 0.00 & 1.12 & 0.57 & 0.39 \\
\hline I170L & 0.00 & 0.01 & 0.02 & 2.31 \\
\hline I170F & 0.00 & 0.03 & 0.05 & 16.48 \\
\hline V181I & 0.00 & 0.05 & 0.00 & -0.73 \\
\hline $\mathrm{T} 206 \mathrm{M}$ & -0.30 & -0.33 & 0.01 & -0.14 \\
\hline G212D & 0.00 & 0.19 & -0.04 & -0.11 \\
\hline Y213H & 0.00 & 0.41 & -0.01 & 1.27 \\
\hline Y214H & 0.00 & -0.01 & 0.00 & 0.18 \\
\hline $\mathrm{T} 216 \mathrm{P}$ & 0.00 & -0.01 & 0.00 & 2.27 \\
\hline $\mathrm{R} 219 \mathrm{~K}$ & 0.00 & 0.00 & 0.00 & 0.00 \\
\hline $\mathrm{A} 222 \mathrm{~V}$ & 0.00 & 0.00 & 0.00 & -0.11 \\
\hline E229K & 0.00 & 0.00 & 0.00 & -0.17 \\
\hline T230A & 0.00 & 0.00 & 0.00 & 0.62 \\
\hline T240N & 0.00 & 0.00 & 0.00 & 0.86 \\
\hline $\mathrm{S} 246 \mathrm{P}$ & 0.00 & 0.00 & 0.00 & -1.11 \\
\hline L254I & 0.00 & 0.00 & 0.00 & 0.81 \\
\hline L254V & 0.00 & 0.00 & 0.00 & 1.39 \\
\hline Q255R & 0.00 & 0.00 & 0.00 & 0.1 \\
\hline $\mathrm{I} 260 \mathrm{R}$ & 0 & 0 & 0 & 1.78 \\
\hline $\mathrm{T} 262 \mathrm{~A}$ & 0 & 0 & 0 & -0.08 \\
\hline W275L & 0 & 0 & 0 & 0.09 \\
\hline A503V & -0.17 & 0.09 & 0 & 0.1 \\
\hline Q508R & 0.16 & -0.03 & 0 & 0.54 \\
\hline Y517C & 0.01 & 0.26 & 0.01 & 1.38 \\
\hline G524D & -0.01 & 0.14 & 2.31 & 2.12 \\
\hline A526T & 0.00 & 0.02 & 0.56 & 0.80 \\
\hline I527T & 0.00 & 0.18 & 0.15 & 1.04 \\
\hline P537L & 0.00 & 0.23 & 0.42 & 0.53 \\
\hline I544T & 0.00 & 0.36 & -0.01 & 0.47 \\
\hline E545D & 0.00 & 0.5 & 0.00 & 0.46 \\
\hline N550K & 4.59 & 0.01 & 0.00 & 0.62 \\
\hline D552N & 1.76 & 0.23 & 0.00 & 0.13 \\
\hline A562D & -0.06 & 2.98 & 0.02 & 0.67 \\
\hline L571R & 0.00 & 0.05 & 2.34 & 0.27 \\
\hline L573R & 0.00 & 2.78 & -0.25 & 1.30 \\
\hline W597F & 0.00 & 0.07 & 0.48 & -0.11 \\
\hline W597C & 0.00 & 0.35 & 3.51 & 0.26 \\
\hline
\end{tabular}

The one observed mutation on the watch list is indicated in red. The two mutations that our methods falsely excludes as non-functional are indicated in blue. All numerical entries are $\Delta \Delta G$ values in units of $\mathrm{kcal} / \mathrm{mol}$. a) Binding affinity between GP and the KZ52 antibody. b) Binding affinity between GP1 and GP2. c) Binding affinity between three GP1-GP2 dimers. d) Folding stability for GP2.

experimental studies that assess how disrupting antibody binding influences immune response.

Davidson et al. (2015) recently conducted an alanine-scanning mutagenesis study on GP that can be qualitatively compared to our work. Specifically, they individually mutated each residue of the GP protein to alanine and measured changes in GP-KZ52 binding affinities relative to the unmutated form. 


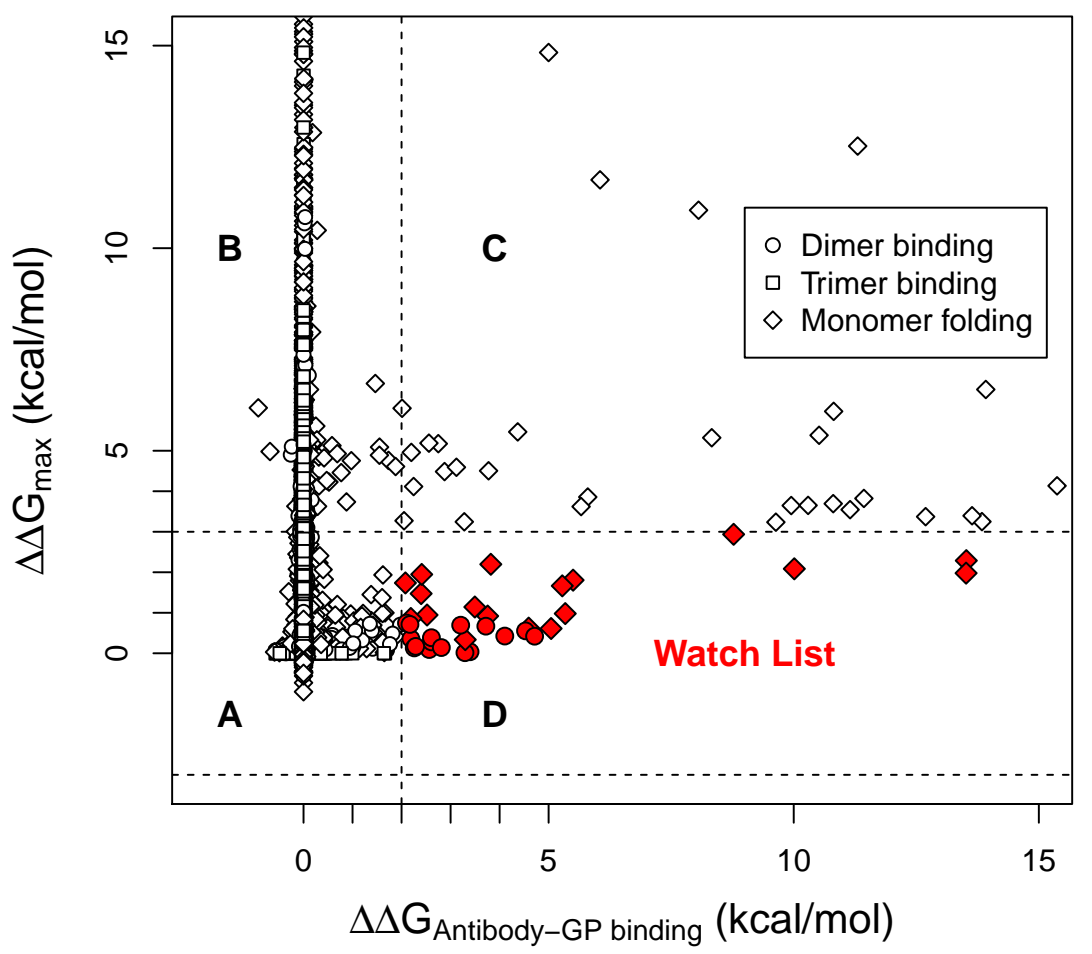

Figure 2. Watch list mutations disrupt KZ52 antibody binding but not GP folding and trimer formation. For each possible GP mutation, only the maximum of folding stability, dimer binding stability (interaction of GP1 and GP2) or trimer binding stability (interaction of a GP1-GP2 dimer with other dimers) is plotted on the y-axis. Symbols in the inset legend indicate which of the three is plotted. The GP-KZ52 binding affinity is plotted on the X-axis. Mutations with X-axis values $-3<\Delta \Delta G<3$ $\mathrm{kcal} / \mathrm{mol}$ are considered functional since they are likely to retain the ability to fold and form trimers (regions A and D). Mutations with y-axis values $\Delta \Delta G>2 \mathrm{kcal} / \mathrm{mol}$ have the potential to disrupt antibody binding (regions $\mathrm{C}$ and $\mathrm{D}$ ). The watch list mutations (region $\mathrm{D}$ ) are those that are likely to be both functional and disrupt antibody binding. The reasoning behind using a different cutoff for functional as compared to antibody binding is described in the main text.

They identified five residues that are critical for KZ52 antibody binding: C511, N550, D552, G553, and C556. Three of these sites are found on our watch list in Table 2 (N550, D552, and G553) and 25 of the $34(74 \%)$ watch list mutations are found at these three sites. For the two other critical residues identified by Davidson et al. (C511 and C556), our results agree that antibody binding is disrupted by mutations at these sites, but we estimate that folding is also disrupted, and hence the exclusion from the watch list. If we ignore our criteria that mutations do not disrupt folding stability or the formation of dimers and trimers, we identify eight residues where at least one mutation will disrupt KZ52 antibody binding: N506, C511, P513, N550, D552, G553, C556, G557 (all individual mutations can be obtained from the spreadsheet in the Supplemental Materials). Overall, we conclude that our results are generally consistent with the findings of (Davidson et al., 2015).

The watch list remains incomplete and putative for several reasons. First, although our list was generated for one EBOV epitope and its interactions with the KZ52 antibody, it is known that there are multiple epitopes (Figure 3). Indeed, a recent study found mutations of a conserved threonine in the EBOV mucin-like domain that is required for protection by the 14G7 antibody (Park et al., 2015). This highlights the need for more experimental structures of antibodies interacting with viral proteins. With more experimental structures, it would be possible to expand the watch list to incorporate more 
epitopes. Second, the watch list only includes substitutions that are predicted to individually disrupt antibody binding while remaining functional. It is alternatively possible that immune escape could arise by the accumulation of several changes, each of modest stability effect but with a large cumulative effect on antibody binding. How multiple substitutions interact to produce cumulative effects on stability is not well understood and is an important consideration for future studies. Third, the watch list has not been experimental validated (except in its general consistency with the work of Davidson et al. (2015)) either in terms of mutational effects on GP folding and binding affinities, nor on the downstream immune system consequences. Our hope is that this work will motivate such research.
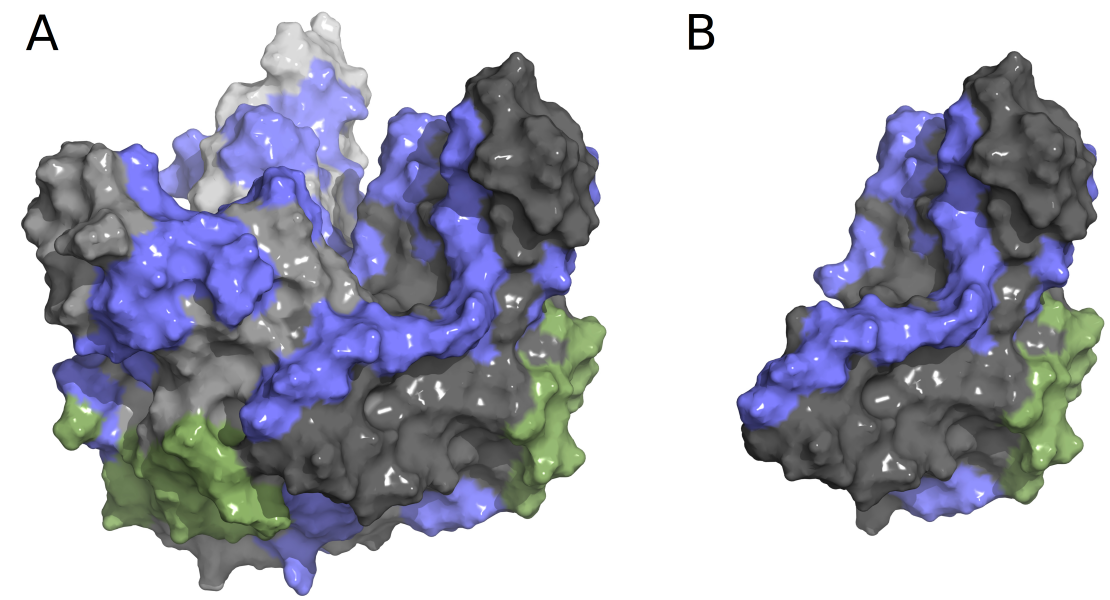

Figure 3. Structure of Ebola GP1-GP2 trimer complex (A) and individual GP1-GP2 dimer (B) with structural epitopes from KZ52 and other known linear epitopes. KZ52 is in green, other known linear epitopes are in blue (Becquart et al., 2014). The watch list generated for the current study is for the green region only, since structures are required for the method used, highlighting the need for more experimental structures of Ebola with antibodies.

In summary, we have initiated a watch list of potential antibody escape mutations of EBOV by considering the interactions between GP and antibody KZ52. This initial watch list contains 34 mutations in six sites in GP2, and one of these mutations (N550K) was seen in humans in a previous outbreak. We believe initiating a watch list is an important first step to predicting how the evolution of EBOV could undermine treatment efforts. Our intention is that the watch list motives experimental research testing the strategy we have employed. This study further emphasizes the need for more experimental structures of antibodies interacting with EBOV in order to produce a comprehensive watch list. We highlight the need for ongoing monitoring of EBOV sequences in human outbreaks. If mutations on the watch list appear in human populations infected by EBOV, treatment with vaccines or antibody therapies may be compromised. Furthermore, if mutations from the watch list arise and increase in frequency within an immunized population, it would suggest that the virus is responding to selective pressure exerted by the vaccine. Monitoring will be much more powerful as the watch list is expanded and experimentally validated. Finally, we suggest that the approach used here is general and could be applied to other viruses for which experimental structures are available. 
Table 2. Watch list mutations and their effects on stability.

\begin{tabular}{|c|c|c|c|}
\hline GP2 mutation ${ }^{a}$ & Antibody bind ${ }^{b}$ & Dimer bind ${ }^{c}$ & Monomer fold ${ }^{d}$ \\
\hline N506W & 3.40 & 0.04 & -0.41 \\
\hline N506Y & 2.56 & 0.09 & -0.55 \\
\hline P513H & 2.52 & 0.01 & 0.95 \\
\hline P513W & 2.19 & 0.01 & 0.86 \\
\hline N550Q & 3.76 & 0.01 & 0.92 \\
\hline N550K & 4.59 & 0.01 & 0.62 \\
\hline N550P & 3.82 & 0.14 & 2.20 \\
\hline N550F & 10.01 & 0.03 & 2.09 \\
\hline $\mathrm{N} 550 \mathrm{H}$ & 5.50 & 0.03 & 1.81 \\
\hline N550I & 5.28 & 0.02 & 1.66 \\
\hline N550E & 3.49 & -0.04 & 1.14 \\
\hline N550R & 5.34 & -0.03 & 0.98 \\
\hline N550W & 13.52 & 0.02 & 2.29 \\
\hline N550V & 2.08 & 0.02 & 1.74 \\
\hline N550Y & 13.52 & 0.04 & 1.98 \\
\hline N550M & 3.29 & 0.02 & -0.15 \\
\hline D552S & 2.10 & 0.75 & 0.33 \\
\hline D552Q & 2.19 & 0.36 & 0.29 \\
\hline D552K & 2.61 & 0.28 & 0.16 \\
\hline D552T & 2.40 & 1.01 & 1.47 \\
\hline D552F & 4.11 & 0.43 & 0.14 \\
\hline D552A & 2.17 & 0.71 & 0.48 \\
\hline D552H & 4.53 & 0.55 & 0.29 \\
\hline D552G & 2.61 & 0.39 & 0.01 \\
\hline D552R & 3.30 & 0.26 & 0.34 \\
\hline D552W & 5.05 & 0.41 & 0.61 \\
\hline D552V & 2.41 & 0.75 & 1.95 \\
\hline D552Y & 4.71 & 0.42 & 0.13 \\
\hline G553M & 8.77 & -0.01 & 2.94 \\
\hline G557F & 2.26 & 0.13 & -1.34 \\
\hline G557H & 3.72 & 0.67 & -0.05 \\
\hline G557R & 2.29 & 0.17 & -0.62 \\
\hline G557W & 3.21 & 0.69 & -1.32 \\
\hline G557Y & 2.81 & 0.14 & -1.19 \\
\hline
\end{tabular}

All numerical entries are $\Delta \Delta G$ values in units of $\mathrm{kcal} / \mathrm{mol}$. a) The 34 mutations are distributed among six sites in GP2. b) Binding affinity between GP and the KZ52 antibody. c) Binding affinity between GP1 and GP2. d) Folding stability for GP2. Binding affinity results for forming the GP trimer are all zero and are not shown. The one observed mutation on the watch list is indicated in red.

\section{REFERENCES}

(2015). Centers for Disease Control. http://www.cdc.gov/vhf/ebola/.

(2015). National Center for Biotechnology Information. http://www.ncbi.nlm.nih.gov/genome/viruses /variation/ebola/.

Arnold, K., Bordoli, L., Kopp, J., and Schwede, T. (2005). The SWISS-MODEL workspace: a web-based environment for protein structure homology modelling. Bioinformatics, 22(2):195-201.

Becquart, P., Mahlakõiv, T., Nkoghe, D., and Leroy, E. M. (2014). Identification of continuous human b-cell epitopes in the VP35, VP40, nucleoprotein and glycoprotein of ebola virus. PLoS ONE, 9(6):e96360.

Borio, L., Cox, E., and Lurie, N. (2015). Combating emerging threats-accelerating the availability of medical therapies. New England Journal of Medicine. 
Brindley, M. A., Hughes, L., Ruiz, A., McCray, P. B., Sanchez, A., Sanders, D. A., and Maury, W. (2007). Ebola virus glycoprotein 1: identification of residues important for binding and postbinding events. Journal of virology, 81(14):7702-7709.

Brister, J. R., Bao, Y., Zhdanov, S. A., Ostapchuck, Y., Chetvernin, V., Kiryutin, B., Zaslavsky, L., Kimelman, M., and Tatusova, T. A. (2013). Virus variation resource-recent updates and future directions. Nucleic acids research, page gkt1268.

Davidson, E., Bryan, C., Fong, R. H., Barnes, T., Pfaff, J. M., Mabila, M., Rucker, J. B., and Doranz, B. J. (2015). Mechanism of binding to ebola virus glycoprotein by the zmapp, zmab, and mb-003 cocktail antibodies. Journal of virology, 89(21):10982-10992.

Dowling, W., Thompson, E., Badger, C., Mellquist, J. L., Garrison, A. R., Smith, J. M., Paragas, J., Hogan, R. J., and Schmaljohn, C. (2007). Influences of glycosylation on antigenicity, immunogenicity, and protective efficacy of ebola virus gp dna vaccines. Journal of virology, 81(4):1821-1837.

Dube, D., Brecher, M. B., Delos, S. E., Rose, S. C., Park, E. W., Schornberg, K. L., Kuhn, J. H., and White, J. M. (2009). The primed ebolavirus glycoprotein (19-kilodalton gp1, 2): sequence and residues critical for host cell binding. Journal of virology, 83(7):2883-2891.

Garbutt, M., Liebscher, R., Wahl-Jensen, V., Jones, S., Möller, P., Wagner, R., Volchkov, V., Klenk, H.-D., Feldmann, H., and Ströher, U. (2004). Properties of replication-competent vesicular stomatitis virus vectors expressing glycoproteins of filoviruses and arenaviruses. Journal of virology, 78(10):54585465.

Geiß, Y. and Dietrich, U. (2014). Catch me if you can-the race between hiv and neutralizing antibodies. AIDS reviews, 17(2):107-113.

Gire, S. K., Goba, A., Andersen, K. G., Sealfon, R. S. G., Park, D. J., Kanneh, L., Jalloh, S., Momoh, M., Fullah, M., Dudas, G., Wohl, S., Moses, L. M., Yozwiak, N. L., Winnicki, S., Matranga, C. B., Malboeuf, C. M., Qu, J., Gladden, A. D., Schaffner, S. F., Yang, X., Jiang, P.-P., Nekoui, M., Colubri, A., Coomber, M. R., Fonnie, M., Moigboi, A., Gbakie, M., Kamara, F. K., Tucker, V., Konuwa, E., Saffa, S., Sellu, J., Jalloh, A. A., Kovoma, A., Koninga, J., Mustapha, I., Kargbo, K., Foday, M., Yillah, M., Kanneh, F., Robert, W., Massally, J. L. B., Chapman, S. B., Bochicchio, J., Murphy, C., Nusbaum, C., Young, S., Birren, B. W., Grant, D. S., Scheiffelin, J. S., Lander, E. S., Happi, C., Gevao, S. M., Gnirke, A., Rambaut, A., Garry, R. F., Khan, S. H., and Sabeti, P. C. (2014). Genomic surveillance elucidates ebola virus origin and transmission during the 2014 outbreak. Science, 345(6202):1369-1372.

Guerois, R., Nielsen, J. E., and Serrano, L. (2002). Predicting changes in the stability of proteins and protein complexes: a study of more than 1000 mutations. Journal of molecular biology, 320(2):369387.

Henao-Restrepo, A. M., Longini, I. M., Egger, M., Dean, N. E., Edmunds, W. J., Camacho, A., Carroll, M. W., Doumbia, M., Draguez, B., Duraffour, S., Enwere, G., Grais, R., Gunther, S., Hossmann, S., Kondé, M. K., Kone, S., Kuisma, E., Levine, M. M., Mandal, S., Norheim, G., Riveros, X., Soumah, A., Trelle, S., Vicari, A. S., Watson, C. H., Kéita, S., Kieny, M. P., and Røttingen, J.-A. (2015). Efficacy and effectiveness of an rvsv-vectored vaccine expressing ebola surface glycoprotein: interim results from the guinea ring vaccination cluster-randomised trial. The Lancet.

Hess, B., Kutzner, C., van der Spoel, D., and Lindahl, E. (2008). GROMACS 4: Algorithms for highly efficient, load-balanced, and scalable molecular simulation. Journal of Chemical Theory and Computation, 4(3):435-447.

Ito, H., Watanabe, S., Sanchez, A., Whitt, M. A., and Kawaoka, Y. (1999). Mutational analysis of the putative fusion domain of ebola virus glycoprotein. Journal of virology, 73(10):8907-8912.

Kajihara, M., Nakayama, E., Marzi, A., Igarashi, M., Feldmann, H., and Takada, A. (2013). Novel mutations in marburg virus glycoprotein associated with viral evasion from antibody mediated immune pressure. Journal of General Virology, 94(Pt 4):876-883.

Kostolanskỳ, F., Varečková, E., Betáková, T., Mucha, V., Russ, G., and Wharton, S. (2000). The strong positive correlation between effective affinity and infectivity neutralization of highly cross-reactive monoclonal antibody iib4, which recognizes antigenic site b on influenza a virus haemagglutinin. Journal of General Virology, 81(7):1727-1735.

Lee, J. E., Fusco, M. L., Hessell, A. J., Oswald, W. B., Burton, D. R., and Saphire, E. O. (2008). Structure of the ebola virus glycoprotein bound to an antibody from a human survivor. Nature, 454(7201):177182.

Lee, K. H., Miller, C. R., Nagel, A. C., Wichman, H. A., Joyce, P., and Ytreberg, F. M. (2011). First-step 
mutations for adaptation at elevated temperature increase capsid stability in a virus. PLoS ONE, 6(9):e25640.

Lennemann, N. J., Walkner, M., Berkebile, A. R., Patel, N., and Maury, W. (2015). The role of conserved $\mathrm{n}$-linked glycans on ebola virus glycoprotein 2. Journal of Infectious Diseases.

Leroy, E. M., Rouquet, P., Formenty, P., Souquière, S., Kilbourne, A., Froment, J.-M., Bermejo, M., Smit, S., Karesh, W., Swanepoel, R., Zaki, S. R., and Rollin, P. E. (2004). Multiple ebola virus transmission events and rapid decline of central african wildlife. Science, 303(5656):387-390.

Maruyama, T., Rodriguez, L. L., Jahrling, P. B., Sanchez, A., Khan, A. S., Nichol, S. T., Peters, C., Parren, P. W., and Burton, D. R. (1999). Ebola virus can be effectively neutralized by antibody produced in natural human infection. Journal of virology, 73(7):6024-6030.

Marzi, A., Engelmann, F., Feldmann, F., Haberthur, K., Shupert, W. L., Brining, D., Scott, D. P., Geisbert, T. W., Kawaoka, Y., Katze, M. G., Feldmann, H., and Messaoudi, I. (2013). Antibodies are necessary for rvsv/zebov-gp-mediated protection against lethal ebola virus challenge in nonhuman primates. Proceedings of the National Academy of Sciences, 110(5):1893-1898.

Miller, C. R., Lee, K. H., Wichman, H. A., and Ytreberg, F. M. (2014). Changing folding and binding stability in a viral coat protein: A comparison between substitutions accessible through mutation and those fixed by natural selection. PLOS ONE, 9(11):e112988.

Olabode, A. S., Jiang, X., Robertson, D. L., and Lovell, S. C. (2015). Ebolavirus is evolving but not changing: No evidence for functional change in EBOV from 1976 to the 2014 outbreak. Virology, 482:202-207.

Oswald, W. B., Geisbert, T. W., Davis, K. J., Geisbert, J. B., Sullivan, N. J., Jahrling, P. B., Parren, P., and Burton, D. R. (2007). Neutralizing antibody fails to impact the course of ebola virus infection in monkeys. PLoS Pathog, 3(1):e9.

Park, D., Dudas, G., Wohl, S., Goba, A., Whitmer, S., Andersen, K., Sealfon, R., Ladner, J., Kugelman, J., Matranga, C., Winnicki, S., Qu, J., Gire, S., Gladden-Young, A., Jalloh, S., Nosamiefan, D., Yozwiak, N., Moses, L., Jiang, P.-P., Lin, A., Schaffner, S., Bird, B., Towner, J., Mamoh, M., Gbakie, M., Kanneh, L., Kargbo, D., Massally, J., Kamara, F., Konuwa, E., Sellu, J., Jalloh, A., Mustapha, I., Foday, M., Yillah, M., Erickson, B., Sealy, T., Blau, D., Paddock, C., Brault, A., Amman, B., Basile, J., Bearden, S., Belser, J., Bergeron, E., Campbell, S., Chakrabarti, A., Dodd, K., Flint, M., Gibbons, A., Goodman, C., Klena, J., McMullan, L., Morgan, L., Russell, B., Salzer, J., Sanchez, A., Wang, D., Jungreis, I., Tomkins-Tinch, C., Kislyuk, A., Lin, M., Chapman, S., MacInnis, B., Matthews, A., Bochicchio, J., Hensley, L., Kuhn, J., Nusbaum, C., Schieffelin, J., Birren, B., Forget, M., Nichol, S., Palacios, G., Ndiaye, D., Happi, C., Gevao, S., Vandi, M., Kargbo, B., Holmes, E., Bedford, T., Gnirke, A., Ströher, U., Rambaut, A., Garry, R., and Sabeti, P. (2015). Ebola virus epidemiology, transmission, and evolution during seven months in sierra leone. Cell, 161(7):1516-1526.

Parren, P. W., Geisbert, T. W., Maruyama, T., Jahrling, P. B., and Burton, D. R. (2002). Pre-and postexposure prophylaxis of ebola virus infection in an animal model by passive transfer of a neutralizing human antibody. Journal of virology, 76(12):6408-6412.

Qiu, X., Alimonti, J. B., Melito, P. L., Fernando, L., Ströher, U., and Jones, S. M. (2011). Characterization of zaire ebolavirus glycoprotein-specific monoclonal antibodies. Clinical immunology, 141(2):218-227.

Qiu, X., Audet, J., Wong, G., Pillet, S., Bello, A., Cabral, T., Strong, J. E., Plummer, F., Corbett, C. R., Alimonti, J. B., and Kobinger, G. P. (2012). Successful treatment of ebola virus-infected cynomolgus macaques with monoclonal antibodies. Science Translational Medicine, 4(138):138ra81-138ra81.

Qiu, X., Wong, G., Audet, J., Bello, A., Fernando, L., Alimonti, J. B., Fausther-Bovendo, H., Wei, H., Aviles, J., Hiatt, E., Johnson, A., Morton, J., Swope, K., Bohorov, O., Bohorova, N., Goodman, C., Kim, D., Pauly, M. H., Velasco, J., Pettitt, J., Olinger, G. G., Whaley, K., Xu, B., Strong, J. E., Zeitlin, L., and Kobinger, G. P. (2014). Reversion of advanced ebola virus disease in nonhuman primates with zmapp. Nature.

Sali, A. and Blundell, T. L. (1993). Comparative protein modelling by satisfaction of spatial restraints. Journal of Molecular Biology, 234(3):779-815.

Schrag, S. J., Rota, P. A., and Bellini, W. J. (1999). Spontaneous mutation rate of measles virus: direct estimation based on mutations conferring monoclonal antibody resistance. Journal of virology, 73(1):51-54.

Schymkowitz, J. W. H., Rousseau, F., Martins, I. C., Ferkinghoff-Borg, J., Stricher, F., and Serrano, L. (2005). Prediction of water and metal binding sites and their affinities by using the fold-x force field. 
Proceedings of the National Academy of Sciences of the United States of America, 102(29):1014710152.

Smith, D. J., Lapedes, A. S., de Jong, J. C., Bestebroer, T. M., Rimmelzwaan, G. F., Osterhaus, A. D., and Fouchier, R. A. (2004). Mapping the antigenic and genetic evolution of influenza virus. Science, 305(5682):371-376.

Stanley, D. A., Honko, A. N., Asiedu, C., Trefry, J. C., Lau-Kilby, A. W., Johnson, J. C., Hensley, L., Ammendola, V., Abbate, A., Grazioli, F., Foulds, K. E., Cheng, C., Wang, L., Donaldson, M. M., Colloca, S., Folgori, A., Roederer, M., Nabel, G. J., Mascola, J., Nicosia, A., Cortese, R., Koup, R. A., and Sullivan, N. J. (2014). Chimpanzee adenovirus vaccine generates acute and durable protective immunity against ebolavirus challenge. Nature medicine.

Tong, Y.-G., Shi, W.-F., Di Liu, Qian, J., Liang, L., Bo, X.-C., Liu, J., Ren, H.-G., Fan, H., Ni, M., Sun, Y., Jin, Y., Teng, Y., Li, Z., Kargbo, D., Dafae, F., Kanu, A., Chen, C.-C., Lan, Z.-H., Jiang, H., Luo, Y., Lu, H.-J., Zhang, X.-G., Yang, F., Hu, Y., Cao, Y.-X., Deng, Y.-Q., Su, H.-X., Sun, Y., Liu, W.-S., Wang, Z., Wang, C.-Y., Bu, Z.-Y., Guo, Z.-D., Zhang, L.-B., Nie, W.-M., Bai, C.-Q., Sun, C.-H., An, X.-P., Xu, P.-S., Zhang, X.-L.-L., Huang, Y., Mi, Z.-Q., Yu, D., Yao, H.-W., Feng, Y., Xia, Z.-P., Zheng, X.-X., Yang, S.-T., Lu, B., Jiang, J.-F., Kargbo, B., He, F.-C., Gao, G. F., Cao, W.-C., Tong, Y.-G., Qian, J., Sun, Y., Lu, H.-J., Zhang, X.-G., Yang, F., Hu, Y., Cao, Y.-X., Deng, Y.-Q., Su, H.-X., Sun, Y., Liu, W.-S., Wang, Z., Wang, C.-Y., Bu, Z.-Y., Guo, Z.-D., Zhang, L.-B., Nie, W.-M., Bai, C.-Q., Sun, C.-H., Feng, Y., Jiang, J.-F., and Gao, G. F. (2015). Genetic diversity and evolutionary dynamics of ebola virus in sierra leone. Nature.

Watanabe, S., Takada, A., Watanabe, T., Ito, H., Kida, H., and Kawaoka, Y. (2000). Functional importance of the coiled-coil of the ebola virus glycoprotein. Journal of virology, 74(21):10194-10201.

Wilson, J. A., Hevey, M., Bakken, R., Guest, S., Bray, M., Schmaljohn, A. L., and Hart, M. K. (2000). Epitopes involved in antibody-mediated protection from ebola virus. Science (New York, N.Y.), 287(5458):1664-1666.

Wittmann, T. J., Biek, R., Hassanin, A., Rouquet, P., Reed, P., Yaba, P., Pourrut, X., Real, L. A., Gonzalez, J.-P., and Leroy, E. M. (2007). Isolates of zaire ebolavirus from wild apes reveal genetic lineage and recombinants. Proceedings of the National Academy of Sciences, 104(43):17123-17127.

Zhan, Y. A. and Ytreberg, F. M. (2015). The cis conformation of proline leads to weaker binding of a p53 peptide to MDM2 compared to trans. Archives of Biochemistry and Biophysics, 575:22-29.

Zhao, X., Liu, E., Chen, F.-P., and Sullender, W. M. (2006). In vitro and in vivo fitness of respiratory syncytial virus monoclonal antibody escape mutants. Journal of virology, 80(23):11651-11657. 